\title{
Linearized Power Flow Equations Based Predictive Control of Transmission Voltages
}

\author{
Mahdi Hajian, Student Member, IEEE, William Rosehart, Senior Member, IEEE, Mevludin Glavic, Senior \\ Member, IEEE, Hamidreza Zareipour, Senior Member, IEEE, and Thierry Van Cutsem, Fellow, IEEE
}

\begin{abstract}
In this paper, an approach based on the concept of Model Predictive Control (MPC) is used to control transmission voltages and prevent long-term voltage instability. The MPC model is based on a linearized steady-state system model derived from power flow equations. Simulation results are presented for the case of Nordic32 test system.
\end{abstract}

Index Terms-Model Predictive Control, Emergency voltage control, Voltage instability, Power flow equations.

\section{NOMENCLATURE}

\begin{tabular}{|c|c|}
\hline$\alpha$ & scaling factor \\
\hline$g b$ & index of generator buses \\
\hline$l b$ & index of load buses \\
\hline$s h$ & $\begin{array}{l}\text { index of load buses where load shedding is } \\
\text { enabled }\end{array}$ \\
\hline $\mathbf{u}$ & vector of control variables \\
\hline & vector of state variables \\
\hline $\mathbf{y}$ & vector of algebraic variables \\
\hline $\mathbf{P}_{\text {sh }}$ & $\begin{array}{l}\text { active power of load buses where load shedding } \\
\text { is possible }\end{array}$ \\
\hline$Q_{g b}$ & generators bus reactive power \\
\hline $\mathrm{S}_{\mathrm{lb}, \mathrm{gb}}$ & $\begin{array}{l}\text { sensitivity matrix of load bus voltages with } \\
\text { respect to generators bus voltages }\end{array}$ \\
\hline $\mathrm{S}_{\mathrm{lb},} \mathbf{P}_{\mathrm{sh}}$ & $\begin{array}{l}\text { sensitivity matrix of load bus voltages with } \\
\text { respect to load shedding }\end{array}$ \\
\hline$q g, g b$ & $\begin{array}{l}\text { sensitivity matrix of generators reactive power } \\
\text { with respect to generators bus voltages }\end{array}$ \\
\hline $\mathbf{S}_{\mathrm{qg}, \mathbf{P}_{\mathrm{sh}}}$ & $\begin{array}{l}\text { sensitivity matrix of generators reactive power } \\
\text { with respect to load shedding }\end{array}$ \\
\hline$T c$ & $\begin{array}{l}\text { the control horizon corresponding to each opti- } \\
\text { mization routine of the MPC }\end{array}$ \\
\hline$T p$ & $\begin{array}{l}\text { the prediction horizon corresponding to each } \\
\text { optimization routine of the MPC }\end{array}$ \\
\hline & generator bus voltages \\
\hline & load bus voltages \\
\hline
\end{tabular}

\section{INTRODUCTION}

M. Hajian, W. Rosehart, and H. Zareipour (e-mail: mhajian, rosehart, h.zareipour@ucalgary.ca) are with the Department of Electrical and Computer Engineering, Schulich School of Engineering, University of Calgary, 2500 University Dr., Calgary, AB, Canada. Mevludin Glavic (glavic@montefiore.ulg.ac.be) is a visiting professor at the Department of Electrical Engineering and Computer Science (Montefiore Institute), University of Liège, Sart Tilman B37, B-4000 Liège, Belgium. Thierry Van Cutsem (t.vancutsem@ulg.ac.be) is with the Fund for Scientific Research (FNRS) and with the same department of the University of Liège.
$\mathbf{V}$ OLTAGE control plays a significant role in system operations given several power system blackouts around the world [1], [2]. The goal in voltage control is to maintain transmission voltages within specified limits while accounting for other system constraints such as generators reactive limits. After a disturbance, power systems might encounter severe drops in bus voltages potentially resulting in voltage instability that need to be corrected [3], [4].

Corrective control of transmission voltages, also named as on-line voltage control, is addressed in several works. In [5], an on-line voltage scheme is presented employing a steady-state model and it is solved via chance constrained optimization. A voltage control model to alleviate voltage violations and minimize transmission losses is presented in [6]. Reference [7] describes a coordinated secondary voltage control, operational in two French control centers, based on a sensitivity model. In [8], a secondary voltage control dealing with emergency actions, such as load shedding, is presented.

Model Predictive Control (MPC) is a class of on-line algorithms which involves sequentially computing a set of future control variables according to received measurements and a system model, applying the first sequence of the computed actions, and repeating the process in the next time step with new collected measurements [9]-[15]. In the power system literature related to MPC for voltage control problems, differential-algebraic equations are often used to model dynamic system responses. Emergency voltage control based on sensitivity analysis calculated via system dynamic equations is addressed in [9]. A tree search optimization approach to coordinate generator voltages, tap changers, and load shedding is presented In [10]. In [11], a coordinated voltage control framework based on non-linear system equations is proposed and solved via Euler state prediction and pseudo gradient evolutionary programming. Reference [12] studies using MPC of load to compute minimum amount of load shedding to restore system voltages. In [13], a centralized quadratic programming to optimally coordinate generator voltage references and load shedding is presented and solved via Lagrangian decomposition. A control switching strategy of shunt capacitors to prevent voltage collapse and maintain a desired stability margin after a contingency is proposed in [14]. In [15], a receding horizon multi-step optimization inspired by MPC is presented to control transmission voltages based on steady-state power flow equations.

In this paper, an MPC-inspired emergency voltage control scheme is presented to correct transmission voltages and prevent long-term voltage instability. Control variables considered 
in this paper are generator voltage setpoints and load shedding. The presented control scheme is based on a linear model in which sensitivities of dependent variables, such as load bus voltages and generators reactive power, to control variables are developed via power flow equations. The actions of Load Tap Changers (LTCs) are implicitly modeled by considering predisturbance load values. Generators reactive power limits are calculated at each MPC time step as a function of active power generation and terminal voltage of machines. The performance of the controller is evaluated in realistic conditions such as monitoring and control issues in which measurement errors exist in the controller scheme. This papers varies from [9][14] in the sense of using a steady-state system model instead of differential-algebraic equations. This paper is also different from [5], [15], where with respect to [5] this paper uses a MPC-based control scheme and a linear model and compared to [15] this paper uses a linear model and a simplified sequence (based on a scaling factor).

The remainder of this paper is structured as follows: General background behind voltage control, MPC, and sensitivities are provided in Section II. In Section III, the proposed control approach is presented. Simulation results obtained using the Nordic32 test system are given in Section IV. Finally, Section $\mathrm{V}$ concludes the paper.

\section{BACKGROUND REVIEW}

\section{A. Voltage Control}

Emergency voltage control is considered in this work, aiming to correct unstable system voltages after a disturbance. Long-term voltage stability is considered in which the actions of LTCs and OverExcitation Limiters (OELs) are mainly involved in long-term system evolution [4]. In the presented controller, it is assumed that snapshots of voltage measurements and generators active power injections are available with a sample period about 5 seconds. This can be achieved in Wide Area Monitoring (WAM) with enhanced measurements provided by Phasor Measurement Units (PMUs) or using SCADA based systems.

The control variables considered include generator voltage setpoints and load curtailment. The problem formulation allows using additional controls, such as transmission line tap changers, active power of generators, and FACTS devices using their steady-state model.

For most of the loads, the amount of active and reactive power depends on system voltages. Therefore, after a large disturbance leading to dramatic voltage drops, the initial consequence is a decrease in system loads. However, by the action of LTCs trying to restore distribution voltages, load values tend to progressively recover to their pre-disturbance values. In the presented approach, pre-disturbance load values are used to implicitly model the action of LTCs.

\section{B. Model Predictive Control}

Generally, MPC involves solving an on-line open loop control optimization problem with a finite horizon subject to the set of equality (system model) and inequality (limits on available controls, rate of changes, etc.) constraints [16].

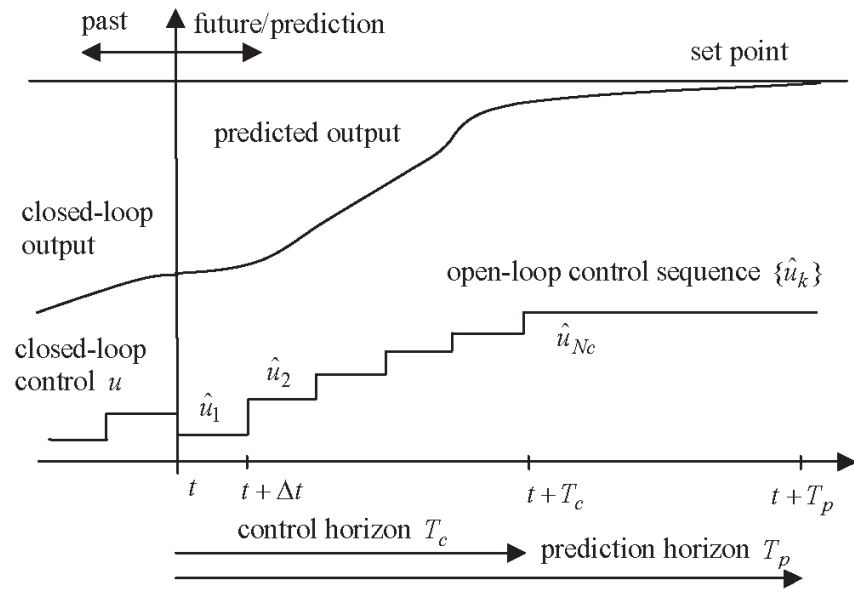

Fig. 1. Basic principle of the MPC [18].

The main feature of the MPC is to solve the optimal control problem in finite time horizon based on on-line analysis [17]. At each time $t$, MPC predicts future system behavior over the prediction horizon, $T p$ and sequentially computes a set of control actions for the system for the control horizon $T c$. The first sequence of the control actions are implemented until the next measurements from the system are available. Then, new controls are calculated based on the updated data from the system. The principle of the MPC is shown in Fig. 1.

\section{Sensitivities}

Sensitivities are used to develop a linear model of system in order to formulate the MPC optimization routine. In this paper, sensitivity matrices are derived via steady-state power flow equations. Given the system to be in equilibrium, the power flow equations can be expressed as:

$$
\phi(\mathbf{u}, \mathbf{y})=\mathbf{0}
$$

Assuming $\boldsymbol{\eta}$ to be a variable of interest, the sensitivity of $\boldsymbol{\eta}$ with respect to $\mathbf{u}$ is given by [4]:

$$
\mathbf{S}_{\boldsymbol{\eta} \mathbf{u}}=-\phi_{\mathbf{u}}^{\mathbf{T}}\left(\phi_{\mathbf{y}}^{\mathbf{T}}\right)^{-1} \nabla_{\mathbf{y}} \boldsymbol{\eta}
$$

where $\phi_{\mathbf{u}}\left(\phi_{\mathbf{y}}\right)$ is the jacobian of $\phi$ with respect to $\mathbf{u}(\mathbf{y})$ and $\nabla_{\mathbf{y}} \boldsymbol{\eta}$ is the gradient of $\boldsymbol{\eta}$ with respect to $\mathbf{y}$. Using the sensitivity matrices calculated from (2), the following relationships are developed:

$$
\begin{aligned}
\Delta \mathrm{V}_{\mathrm{lb}} & =\mathrm{S}_{\mathrm{lb}, \mathrm{gb}} \Delta \mathrm{V}_{\mathrm{gb}}+\mathrm{S}_{\mathrm{lb}, \mathrm{P}_{\mathrm{sh}}} \Delta \mathrm{P}_{\mathrm{sh}} \\
\Delta \mathrm{Q}_{\mathrm{gb}} & =\mathrm{S}_{\mathrm{qg}, \mathrm{gb}} \Delta \mathrm{V}_{\mathrm{gb}}+\mathrm{S}_{\mathrm{qg}, \mathrm{P}_{\mathrm{sh}}} \Delta \mathrm{P}_{\mathrm{sh}}
\end{aligned}
$$

where the matrices $\mathbf{S}$, as defined in the Nomenclature, give an approximate linear relationship between different variables in the system, and $\Delta$ shows a change in the corresponding variables. In particular, (3a) provides a relationship between changes in the voltage magnitude at load buses as a function of changes in generator voltage magnitudes, $\Delta \mathbf{V}_{\mathbf{g b}}$, and load shedding, $\Delta \mathbf{P}_{s h}$, in steady state conditions. Similarly, (3b), provides a relationship between changes in generators reactive power and the control variables. 
III. Power Flow Based Model Predictive Control

The proposed MPC model involves the following:

1) at a given time step collect measurements;

2) compute the controls that should be applied at the next time step to bring all voltages and generators reactive power in the desired intervals;

3 ) at the next step, apply a scaled value of the computed sequence;

4) repeat steps 1 to 3 .

In this paper to preserve simplicity in the formulation, control actions are computed for one time step only. The raw values obtained from the solution of the MPC are scaled using a scalar constant $\alpha$, i.e.:

$$
\begin{aligned}
& \mathbf{V}_{\mathbf{g b}}=\mathbf{V}_{\mathbf{g b}}+\alpha \boldsymbol{\Delta} \mathbf{V}_{\mathbf{g b}} \\
& \mathbf{P}_{\mathbf{s h}}=\mathbf{P}_{\mathbf{s h}}-\alpha \boldsymbol{\Delta} \mathbf{P}_{\mathbf{s h}}
\end{aligned}
$$

The value of $\alpha$ is between 0 and 1; however, choosing a proper value is a compromise between smooth system response and effective actions. Large values of $\alpha$ would result in large oscillations in the system, while small values might result in ineffective control action that are not unable to provide sufficient actions to save the system from the voltage collapse. Reference [7] also used a scaling factor in order to apply computed controls progressively and smoothly. However, the formulation used in this paper is different as $\alpha$ is applied to the solution of the whole optimization problem while in [7] it is involved in the objective to define the amount of voltage corrections. Also, the emphasis here is on emergency control including unstable voltage conditions (as demonstrated in Section IV), a situation not dealt with in [7].

The MPC optimization routine can be formulated as follows:

$$
\begin{array}{ll} 
& \min \sum_{s h} \Delta \mathbf{P}_{\mathrm{sh}} \\
\text { s.t. } & \Delta \mathbf{V}_{\mathrm{gb}, \mathrm{lb}}^{\min } \leq \Delta \mathbf{V}_{\mathrm{gb}, \mathrm{lb}} \leq \Delta \mathrm{V}_{\mathrm{gb}, \mathrm{lb}}^{\max } \\
& \Delta \mathbf{Q}_{\mathrm{gb}}^{\min } \leq \Delta \mathbf{Q}_{\mathrm{gb}} \leq \Delta \mathbf{Q}_{\mathrm{gb}}^{\max } \\
& \mathbf{0} \leq \Delta \mathbf{P}_{\mathrm{sh}} \leq \Delta \mathbf{P}_{\mathrm{sh}}^{\max } \\
& \Delta \mathbf{V}_{\mathrm{lb}}=\mathbf{S}_{\mathrm{lb}, \mathrm{gb}} \Delta \mathbf{V}_{\mathrm{gb}}+\mathbf{S}_{\mathrm{lb}, \mathbf{P}_{\mathrm{sh}}} \Delta \mathbf{P}_{\mathrm{sh}} \\
& \Delta \mathbf{Q}_{\mathrm{gb}}=\mathbf{S}_{\mathrm{qg}, \mathrm{gb}} \Delta \mathbf{V}_{\mathrm{gb}}+\mathbf{S}_{\mathrm{qg}, \mathbf{P}_{\mathrm{sh}}} \Delta \mathbf{P}_{\mathrm{sh}}
\end{array}
$$

The objective function of the linear programming described in (5) is defined to minimize the amount of curtailed load at buses where load shedding is allowed while satisfying voltage and reactive constraints.

The constraints (5b)-(5c) describes the lower and upper bounds over the control and state variables of the system. The constraint (5e)-(5f) refers to the sensitivity constraints described in (3a)- (3b).

Note that, in (5) at each time step with new measurement collected from the system, the sensitivity matrices and reactive power limits are updated. The controller computes new control actions and implements scaled values of them, which is called first control sequence in the MPC theory. In (5), load reactive power is not directly involved; instead the controller sheds load reactive power with the same ratio as load active power such that constant power factor is preserved.

The following remarks need to be addressed regarding the control scheme.

\section{A. Load Power Restoration}

As mentioned in Section II-A, in order to model the actions of LTCs in load restoration, loads are set to their pre-disturbance values. As shown in [15], considering LTCs actions in this manner alleviates the need for using complex load models while leading to a satisfactory voltage control with minimal information about the load behavior.

\section{B. Controller Activation}

The controller collects measurements of voltage and bus power injections at a 5-second sample period. The controller is not activated while all bus voltage magnitudes and reactive power generations are within limits. After a detection of violated voltages or generators reactive power, the controller calculates required changes to bring all variables back to acceptable boundaries. Changes in the network topology after the disturbance (if any) are also reflected in the controller. To accommodate computational time and communication delays between the controller and generator/load sites, a 5-second delay is considered between computation of control actions and their implementation on the system.

\section{Generator Voltage Setpoints}

There is normally a steady-state error between the terminal voltages of generators and their Automatic Voltage Regulator (AVR) setpoint [4]. In (5), the terminal voltage of machines is involved in the optimization, while the controller needs to determine new AVR setpoints of generators. In order to deal with this issue, it is known by experience that changes in AVR setpoints of machines result in almost the same change in their terminal voltages [15]. Hence, the controller provides setpoint corrections of AVRs based on computed changes in terminal voltages.

\section{Generators Reactive Power Limits}

Following generator capability curves, the reactive power limit of each generator is a function of its associated active power generation and terminal voltage. The reactive power limits are calculated using collected measurements from the system and equations (3.32a, 3.32b, and 3.49) from [4] where the effect of saturation is omitted for the sake of simplicity.

The other important point that should be taken into consideration is the activation of OELs since they prevent generators from increasing their voltages. However, in practice, it is unlikely to have the information about the action of OELs in the control center. Instead, by using the snapshot of generators reactive power, it is likely that OELs are activated when the reactive power of a machine is equal or higher than its maximum calculated value. Therefore, in the MPC routine, 
when there is a maximum reactive power violation, no increase is possible in the machine voltage. In this way, it is ensured that the machine will be still under AVR control.

\section{Numerical Simulation AND AnAlysis}

\section{A. Test system and Data}

Simulations were carried out using Nordic32 test system [19] shown in Fig. 2. The system consists of 52 buses including 20 voltage controlled buses with synchronous machines (denoted as $g$, followed by machine number). A detailed dynamic model of generators, AVRs, governors, and load is developed in order to utilize the controller in realistic conditions. Each synchronous machine is modeled using a standard synchronous machine model with 3 or 4 rotor windings, a simple governor for generations participating in frequency control, and a simple AVR model including OEL. The loads are represented by an exponential model with exponent 1(constant current) for the active power and exponent 2 (constant admittance) for the reactive power. Each load is supplied via an LTC in which there is a 30 second delay on the first tap change and a shorter delay on subsequent steps. The total amounts of active and reactive load in the system are 10940 MW and 3689 MVAR, respectively. The model was run with MATLAB/SIMULINK tool detailed in [20] using variable step size method to simulate its dynamics, and the optimization problem was solved using TOMLAB with the LP solver interfaced with MATLAB [21]. The simulations were performed on a Mac machine equipped with Intel $2.80 \mathrm{GHz}$ Core 2 Duo CPU and 4 GB of RAM. The proposed controller is able to change 20 generator voltages in the range [0.95 $1.07] \mathrm{pu}$, and the loads at buses 1022, 1041, 1042, 1043, 1044, 1045 , and 2031.

\section{B. Disturbance Scenario}

The considered disturbance is the outage of transmission line $4032-4044$ (Fig. 2) which happens at $t=20 \mathrm{~s}$. Due to the actions of LTCs trying to restore distribution voltages, and the field current limitation of g6, g7, g11, g12, g13, g14, g15, and $\mathrm{g} 16$ the system collapse happens at $t=142 \mathrm{~s}$. The evolution of three load bus voltages are shown in Fig. 3.

\section{MPC Controller}

Upon the detection of violated voltages, the controller becomes activated at $t=65 \mathrm{~s}$ and starts issuing control actions. The value of $\alpha$ is selected to be 0.3 to obtain smooth and effective controls. The controller stabilizes the system by adjusting generator voltages and shedding $183 \mathrm{MW}$ load. Fig. 4 shows the voltage profile of three load bus with the MPC controller.

\section{Impact of Varying the Scaling Factor}

The effect of changing the scaling factor, $\alpha$, over the amount of curtailed load is shown in Table I. The low value of $\alpha(0.1)$ results in implemented actions on the system being ineffective and eventually the system collapses. With increasing the value of $\alpha$, the amount of curtailed load is increased and the system

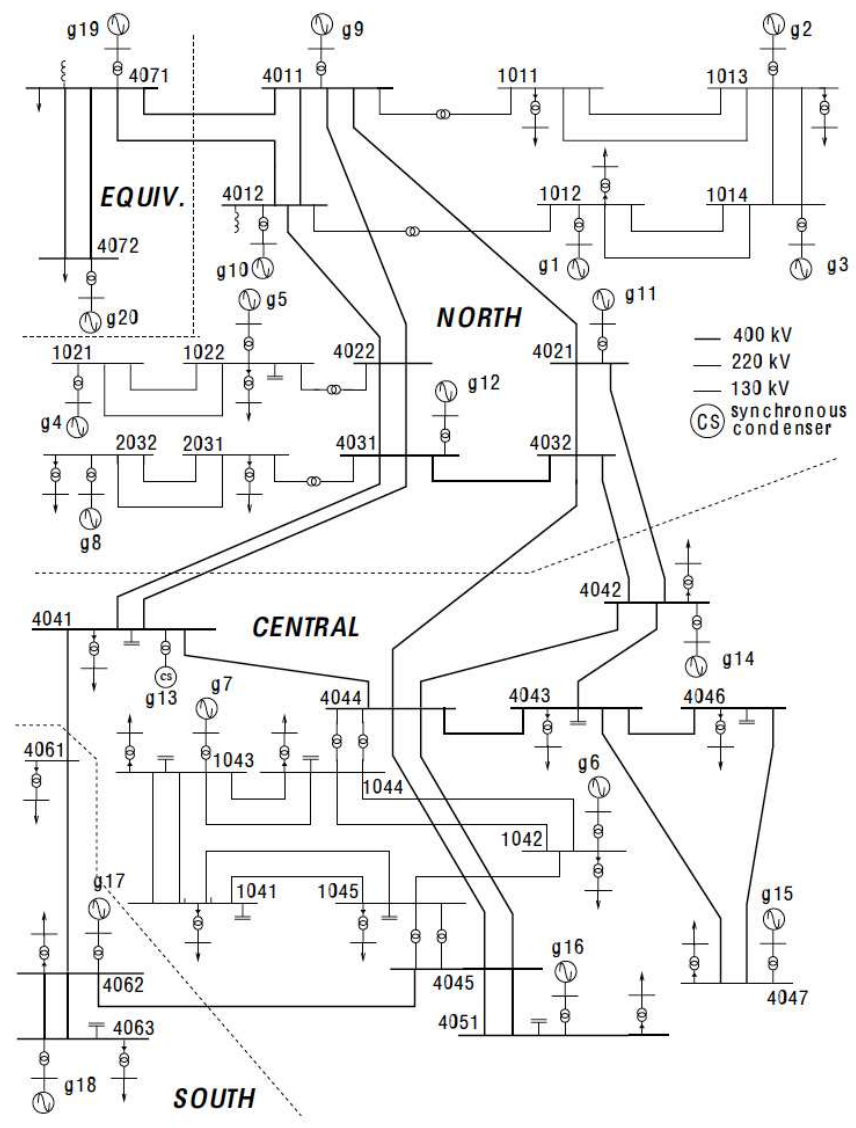

Fig. 2. Single-line diagram of Nordic32 test system [19].

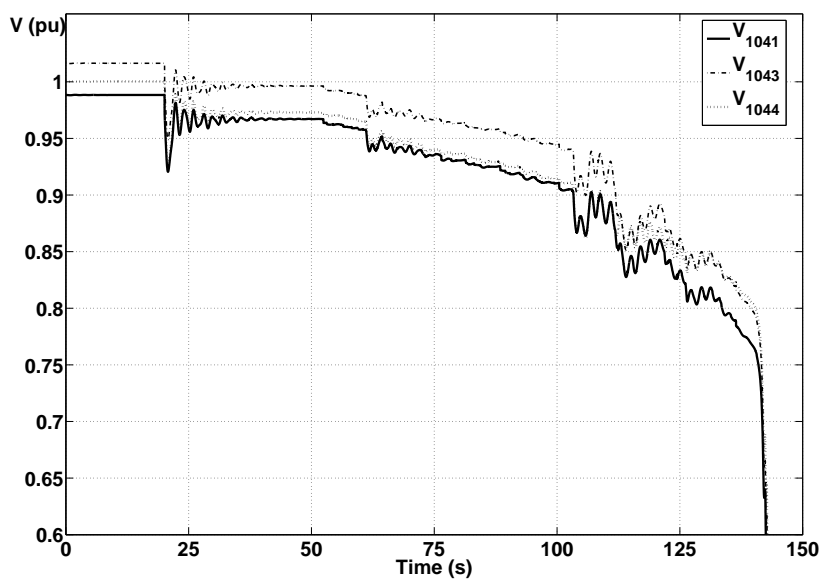

Fig. 3. The evolution of three bus voltages after the disturbance without MPC.

stabilizes with a higher speed. However, high values of alpha might result in temporary oscillations due to high amount of changes suddenly implemented on the system.

\section{E. Reactive Power Limits and OEL Actions}

As described in Section III-D, reactive power limits of synchronous machines depend on active power generation and terminal voltages. In the controller, the reactive power limits 


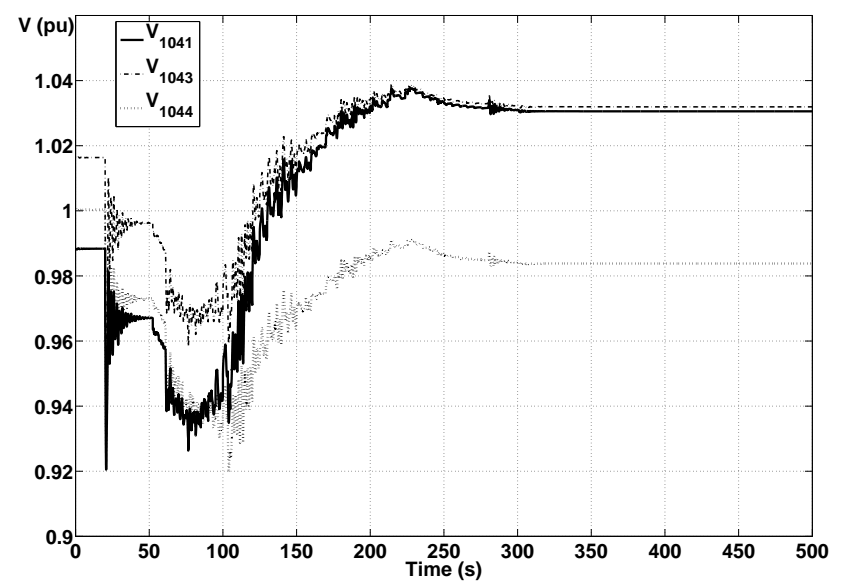

Fig. 4. The evolution of three bus voltages after the disturbance with MPC.

TABLE I

THE AMOUNT OF CURTAILED LOAD WITH RESPECT TO THE SCALING FACTOR.

\begin{tabular}{|c|c|}
\hline Scaling factor $(\alpha)$ & Load shedding (MW) \\
\hline 0.1 & SC \\
\hline 0.2 & 181 \\
\hline 0.3 & 183 \\
\hline 0.4 & 189 \\
\hline 0.5 & 198 \\
\hline 0.6 & 209 \\
\hline 0.7 & 223 \\
\hline 0.8 & 236 \\
\hline 0.9 & 243 \\
\hline 1 & 258 \\
\hline
\end{tabular}

SC: System Collapse

of generators are calculated and updated at each step time. In order to demonstrate the significance of this matter, a simulation was carried out in which static limits of synchronous machines were used in the optimization scheme. The controller was not able to stabilize the system and system collapses at $t=177 \mathrm{~s}$. Figure 5 (a) shows reactive power, the static reactive limit and so-called dynamic reactive limit of g14. The dynamic reactive limit of g14 is less than its static limit used in the controller. Anticipating the reactive power capacity on g14, the controller aims to increase the AVR setpoint as shown in Fig. 5(b) which is not successful since the OEL is activated on g14 at $t=80 \mathrm{~s}$. As a result of this issue on g14 and similar generators, control actions of the controller are not effective and the system collapses.

Another important aspect in the controller is the consideration of OEL actions. As a result of the OEL action, a generator no longer responds to increasing AVR signals requested by the controller which may result in controller failure. In a set of simulations, the impact of OEL actions was not considered in the controller, i.e. generators that have reached their maximum reactive power limit were allowed to increase their AVR

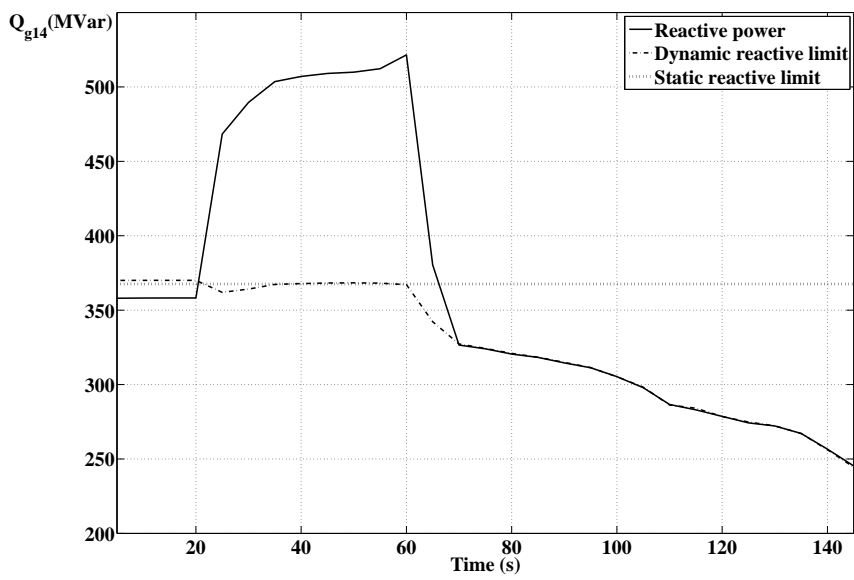

(a) Reactive power, static limit and dynamic limit on g14.

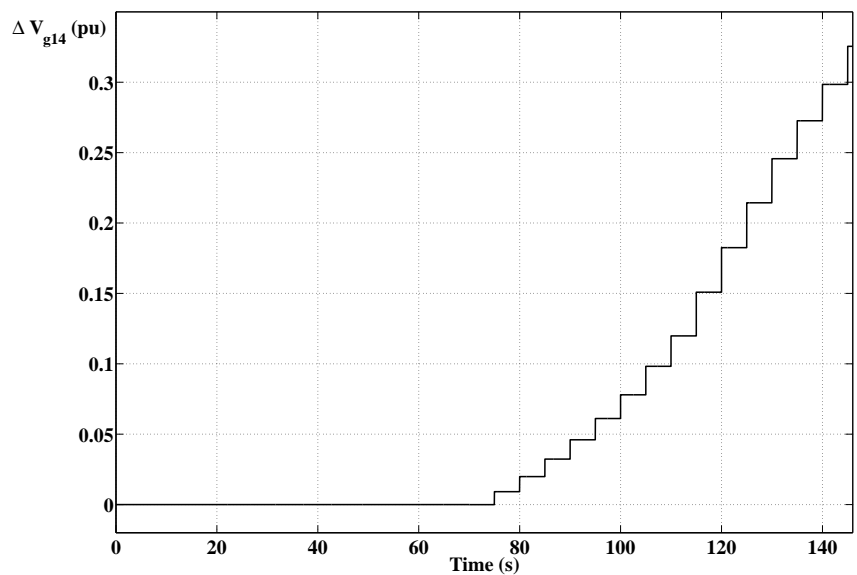

(b) AVR changes on g14 requested by the controller.

Fig. 5. Reactive power (a) and AVR changes (b) on g14 by considering static reactive limits.

setpoints in the optimization scheme. As a result, the system collapsed at $t=155 \mathrm{~s}$. Note that, the controller is aware that the maximum reactive power limits of generators near the disturbance area are reached. However, the controller tries to increase the voltage setpoints of these machines to provide voltage support to the disturbance area and at the same time it tries to decrease the voltage setpoints of other generators in order to keep reactive power of violated generators at their maximum limit. Figure 6 shows this condition on g14 in which the controller increases the AVR setpoint while the machine is at maximum reactive power limit. Since the OEL of g14 is activated on $t=70 \mathrm{~s}$, the increasing signal is not responded by the machine and the system eventually collapses.

By considering the OEL actions the controller does not allow any increase in the AVR setpoints of machines exceeding their maximum limit. The impact of anticipating the OEL actions in the controller on g14 is shown in Fig. 7. By implementing decreasing voltage signals, the controller ultimately releases g14 from its OEL limitation at $t=230 \mathrm{~s}$. 
TABLE II

THE IMPACT OF ERRORS IN MEASUREMENTS AND SYSTEM MODEL ON THE CONTROLLER PERFORMANCE.

\begin{tabular}{|c|c|c|c|c|c|c|}
\cline { 2 - 7 } \multicolumn{1}{c|}{} & \multicolumn{2}{c|}{$10 \%$ error in Ybus } & \multicolumn{2}{c|}{$10 \%$ error in measured voltages } & \multicolumn{2}{c|}{$5 \%$ error in Ybus and measured voltages } \\
\cline { 2 - 7 } & $\begin{array}{c}\text { Percentage of success } \\
\text { in system saving }\end{array}$ & $\begin{array}{c}\text { Average amount of } \\
\text { load shedding }\end{array}$ & $\begin{array}{c}\text { Percentage of success } \\
\text { in system saving }\end{array}$ & $\begin{array}{c}\text { Average amount of } \\
\text { load shedding }\end{array}$ & $\begin{array}{c}\text { Percentage of success } \\
\text { in system saving }\end{array}$ & $\begin{array}{c}\text { Average amount of } \\
\text { load shedding }\end{array}$ \\
\hline Performance & 77 & 1.88 & 92 & 2.59 & 90 & 2.23 \\
\hline
\end{tabular}

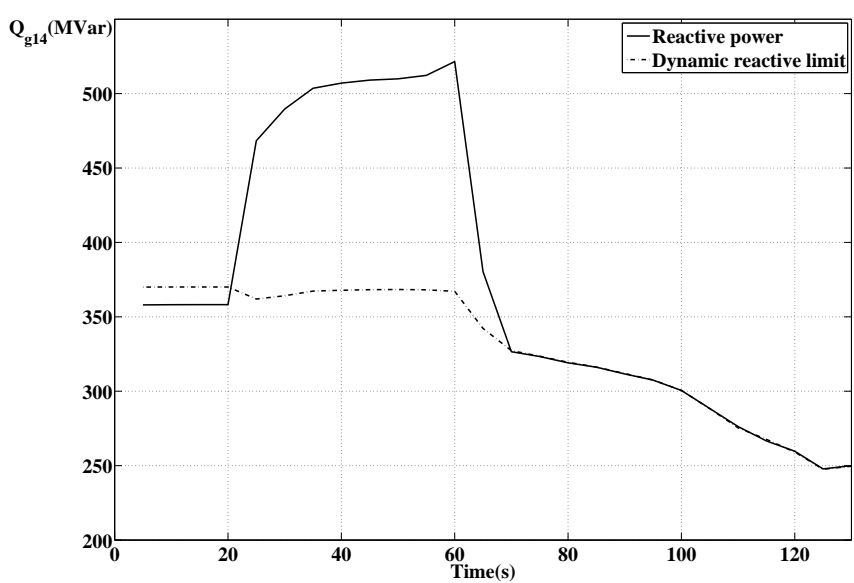

(a) Reactive power and dynamic reactive power limit on g14.

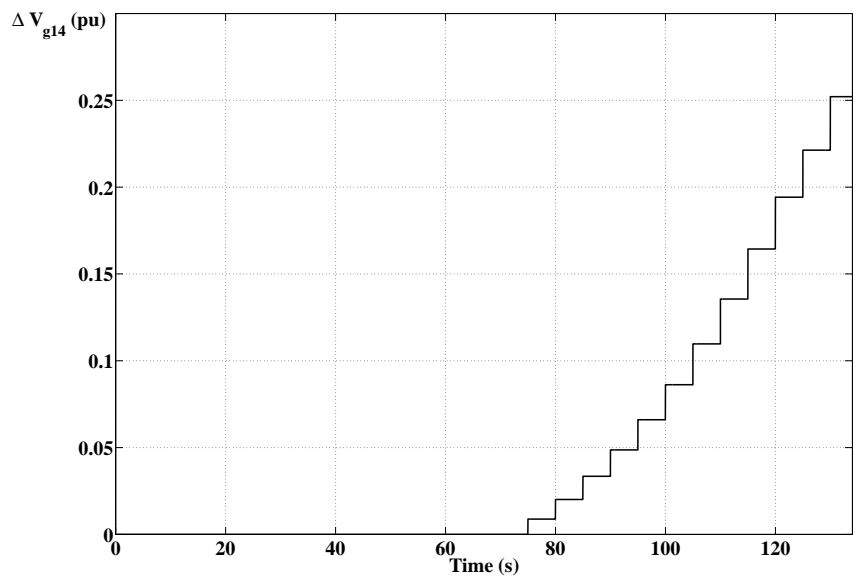

(b) AVR changes on g14 requested by the controller.

Fig. 6. Reactive power (a) and AVR changes (b) on g14 without the consideration of OEL action.

\section{F. The effect of uncertainty in measurements and system model on the MPC performance}

The impact of measurement and modeling errors on the controller performance is considered in this Section. To simulate this situation, normally distributed random noises are introduced to measured voltages and admittance matrix used in the controller scheme. Table. II shows average results of considering errors after 100 runs of the simulations. The considered errors are: $10 \%$ maximum error in the impedance of each transmission line (modeled by modifying the system Ybus), $10 \%$ error in measured voltages due to measurement

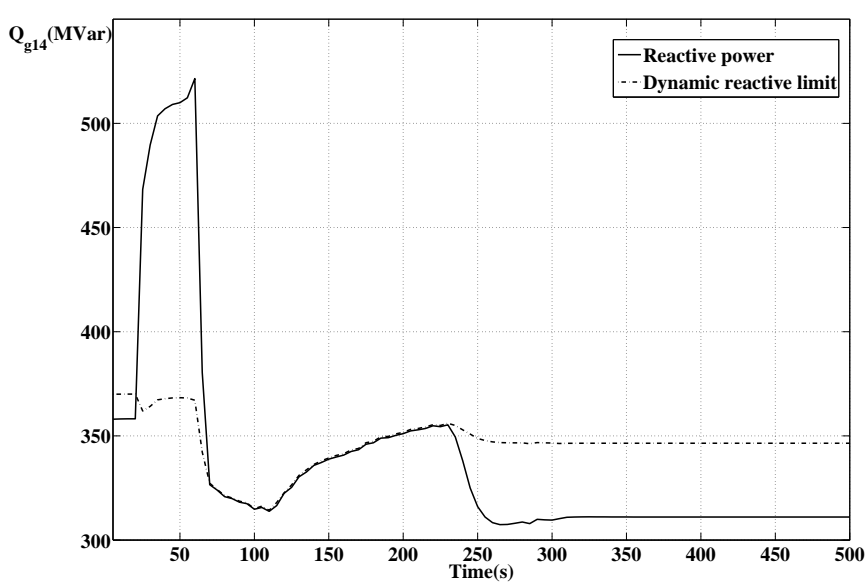

(a) Reactive power and dynamic reactive power limit on g14.

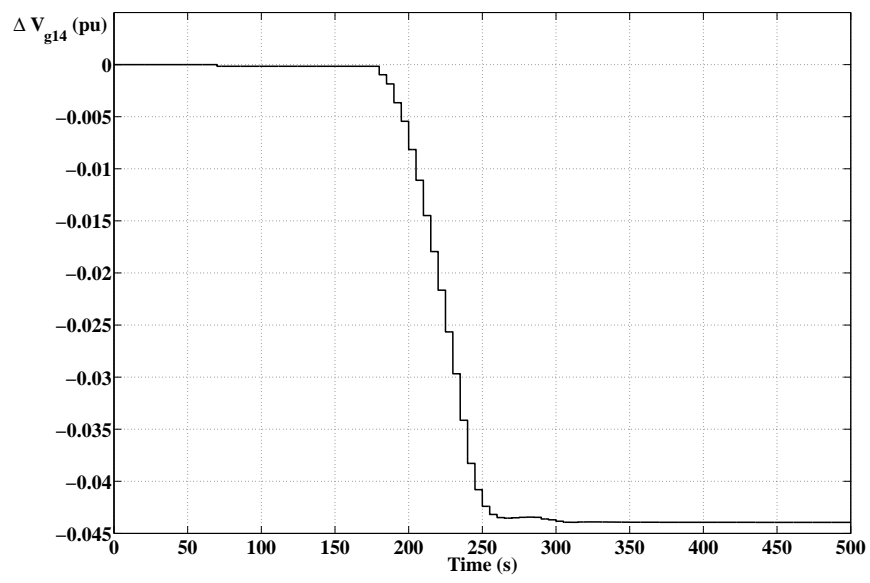

(b) AVR changes on g14 requested by the controller.

Fig. 7. Reactive power (a) and AVR changes (b) on g14 with the consideration of OEL action.

noises, $5 \%$ error in Ybus and measured voltages. As can be observed, although no uncertainty is directly considered in the controller scheme, the controller is successful in saving the system in most of the time. The closed-loop nature of the proposed control offers inherent robustness with respect to modeling and measurement uncertainties.

\section{CONCLUSION}

In this paper, focusing on long-term voltage stability, a steady-state linear MPC-based model was proposed to control transmission voltages. Sensitivity matrices based on power flow equations were employed to develop a linear system 
model. Dynamic reactive limits of synchronous machines were incorporated to effectively model operating limits of generators. The impact of uncertainty in measurements and modeling errors on the controller performance was also investigated.

\section{REFERENCES}

[1] "Final report on the august 14, 2003 blackout in the united states and canada: Causes and recommendations," US-Canada Power System Outage Task Force, 2004, accessed in June 2010. [Online]. Available: https://reports.energy.gov/

[2] "Final report: System disturbance on 4 november, 2006," Union for the Co-ordination of Transmission of Electricity, 2007, accessed in June 2010. [Online]. Available: http://www.entsoe.eu/fileadmin/user_upload/ _library/publications/ce/otherreports

[3] C. W. Taylor, Power system voltage stability. New York: McGraw-Hill, 1994.

[4] T. Van Cutsem and C. Vournas, Voltage Stability of Electric Power Systems. Boston: Kluwer, 1998.

[5] M. Hajian, M. Glavic, W. Rosehart, and H. Zareipour, "A chanceconstrained optimization approach for control of transmission voltages," IEEE Trans. Power Syst., vol. 27, no. 3, pp. 1568 - 1576, Aug. 2012.

[6] S.-K. Chang, F. Albuyeh, M. Gilles, G. Marks, and K. Kato, "Optimal real-time voltage control," IEEE Trans. Power Syst., vol. 5, no. 3, pp. 750 - 8, Aug. 1990.

[7] H. Vu, P. Pruvot, C. Launay, and Y. Harmand, "An improved voltage control on large-scale power system," IEEE Trans. Power Syst., vol. 11, no. 3, pp. 1295 - 303, 1996.

[8] D. Popovic, V. Levi, and Z. Gorecan, "Co-ordination of emergency secondary-voltage control and load shedding to prevent voltage instability," IEE Proceedings-Generation, Transmission and Distribution, vol 144, no. 3, pp. 293 - 300, 1997.

[9] M. Zima and G. Andersson, "Stability assessment and emergency control method using trajectory sensitivities," 2003 IEEE Bologna PowerTech, vol. 2, 2003.

[10] M. Larsson and D. Karlsson, "Coordinated system protection scheme against voltage collapse using heuristic search and predictive control," IEEE Trans. Power Syst., vol. 18, no. 3, pp. 1001 - 6, 2003.

[11] J. Wen, Q. Wu, D. Turner, S. Cheng, and J. Fitch, "Optimal coordinated voltage control for power system voltage stability," IEEE Trans. Power Syst., vol. 19, no. 2, pp. 1115 - 22, 2004.

[12] I. Hiskens and B. Gong, "Voltage stability enhancement via model predictive control of load," Bulk Power System Dynamics and Control VI, pp. 117 - 124, 2004.

[13] A. Beccuti, T. Demiray, G. Andersson, and M. Morari, "A lagrangian decomposition algorithm for optimal emergency voltage control," IEEE Trans. Power Syst., vol. 25, no. 4, pp. 1769 - 1779, 2010.

[14] L. Jin, R. Kumar, and N. Elia, "Model predictive control-based real-time power system protection schemes," IEEE Trans. Power Syst., vol. 25, no. 2, pp. 988 - 998, 2010.

[15] M. Glavic, M. Hajian, W. Rosehart, and T. Van Cutsem, "Recedinghorizon multi-step optimization to correct nonviable or unstable transmission voltages," IEEE Trans. Power Syst., vol. 26, no. 3, pp. 16411650, Aug. 2011.

[16] J. M. Maciejowski, Predictive Control with Constraints. Prentice Hall, 2002.

[17] D. Mayne, J. Rawlings, C. Rao, and P. Scokaert, "Constrained model predictive control: stability and optimality," Automatica, vol. 36, no. 6, pp. $789-814$, Jun. 2000

[18] M. Glavic and T. Van Cutsem, "Some reflections on model predictive control of transmission voltages," 2006 38th Annual North American Power Symposium, NAPS-2006 Proceedings, pp. 625 - 632, Sep. 2006.

[19] — "Wide-area detection of voltage instability from synchronized phasor measurements. part II: Simulation results," IEEE Trans. Power Syst., vol. 24, no. 3, pp. 1417 - 1425, 2009.

[20] C. Vournas, E. Potamianakis, C. Moors, and T. Van Cutsem, "An educational simulation tool for power system control and stability," IEEE Trans. Power Syst., vol. 19, no. 1, pp. 48 - 55, 2004.

[21] "A general purpose, open matlab environment for research and teaching in optimization." [Online]. Available: http://tomopt.com/ 